\title{
The stochastic programming heritage of Maarten van der Vlerk
}

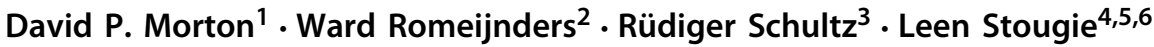

On October 9, 2016, Professor Maarten H. van der Vlerk died tragically. This special issue is dedicated to his memory.

Published online: 6 September 2018

(c) Springer-Verlag GmbH Germany, part of Springer Nature 2018

Maarten van der Vlerk was born in the year 1961 in Assen, The Netherlands, where he spent his childhood and attended school up to the A-level, confirming the requisite maturity for university studies. At the University of Groningen he became a student in the Department of Econometrics and commenced his doctoral studies there under the supervision of Wim Klein Haneveld and Leen Stougie in 1990. Invited and encouraged by his supervisors, Maarten placed a research focus on the integer programming side of stochastic optimization. Soon, he discovered his first original results culminating in a 1993 paper in Mathematical Programming with François Louveaux of the Facultés Universitaires Notre Dame de la Paix in Namur, on "Stochastic programming with simple integer recourse" (Louveaux and van der Vlerk 1993). With his thesis "Stochastic programming with integer recourse", Maarten was awarded his Ph.D. degree in Economics from the University of Groningen in 1995 (van der Vlerk 1995).

After a year as postdoc at the Center for Operations Research and Econometrics (CORE) at the Université Catholique de Louvain at Louvain-la-Neuve, Maarten returned to Groningen. In 1999 he received a prestigious research fellowship from the Royal Netherlands Academy of Sciences. In 2008, Maarten became Professor of Stochastic Optimization at the University of Groningen, Faculty of Economics and Business, Department of Operations.

From the mid 1990s on, stochastic integer programming - with its structures to be detected, its algorithms to be constructed, and, last but not least, its real-life applications to be developed-became the field where Maarten van der Vlerk's research earned highest recognition in the stochastic programming community and beyond. A permanently recurring research target of Maarten, and his various coauthors, has been convex approximation of the notoriously non-convex, discontinuous, mixed-integer

Rüdiger Schultz

ruediger.schultz@uni-due.de

Extended author information available on the last page of the article 
expected recourse function, together with its algorithmic utilization. Papers devoted to these topics range over a time span of more than 20 years.

Maarten's publications on structures began with "On the convex hull of the simple integer recourse objective function" jointly with Wim Klein Haneveld and Leen Stougie in Annals of Operations Research in 1995 (Klein Haneveld et al. 1995). His work on structures continued with "Convex approximations for complete integer recourse models", Mathematical Programming (2004) (van der Vlerk 2004), and "Simple integer recourse models: convexity and convex approximations", Mathematical Programming (2006) (Klein Haneveld et al. 2006), the latter again jointly with Wim Klein Haneveld and Leen Stougie.

Our understanding of the structure of integer recourse problems received another boost with Maarten's 2015 paper in SIAM Journal on Optimization, "Convex approximations for totally unimodular integer recourse models: a uniform error bound" (Romeijnders et al. 2015), written together with Wim Klein Haneveld and their coadvised doctoral student, Ward Romeijnders. Departure points for these investigations involve periodicity properties of mixed-integer value functions and their interplay with the total variation of density functions. This line of research-extended with journal publications (Romeijnders et al. 2016a, b) — continues ever since and, in fact, leads into the present special issue, whose first article has been coauthored by Maarten van der Vlerk.

Along with structural investigation, Maarten always had an eye on algorithms and computations. Already in a 1996 paper, "An algorithm for the construction of the convex hull of the simple integer recourse objective function" (Klein Haneveld et al. 1996) in Annals of Operations Research, jointly with Wim Klein Haneveld and Leen Stougie, an algorithm is proposed for finding the convex hull, i.e., tight lower bounds, for the objective function. Detection of convex hulls for recourse functionals has always been accompanied by numerical testing. A 2017 paper jointly with David Morton and Ward Romeijnders in INFORMS Journal on Computing is an example of the latter.

In 1998, Maarten, jointly with Rüdiger Schultz and Leen Stougie, published in Mathematical Programing an implicit-enumeration algorithm for two-stage stochastic integer programs, where function value calculation is enhanced by techniques from Gröbner bases in computational algebra (Schultz et al. 1998). This work initiated considerable subsequent research on primal augmentation in stochastic programming.

Another topic Maarten dealt with, often in collaboration with Wim Klein Haneveld, concerns integrated chance constraints. In a 2006 joint paper (Klein Haneveld and van der Vlerk 2006), they analyze structures and propose algorithms for numerically handling these instruments for risk aversion.

Today, almost any decision to be taken with an aspiration for being "best" is exposed to uncertainty, and stochastic optimization is increasingly the method of choice for practical planning under uncertainty. This is reflected by the variety of practical applications Maarten van der Vlerk worked on. To list a few, we start with telecommunications, or more precisely, distributed processing in telecommunication networks (Tomasgard et al. 1998), with Asgeir Tomasgard, Jan A. Audestad, Shane Dye, Leen Stougie, and Stein W. Wallace. The list continues with generalized assignment problems (Albareda-Sambola et al. 2006), with Maria Albareda-Sambola and 
Elena Fernández, day-ahead paratransit planning (Cremers et al. 2009), with Maria L.A.G. Cremers and Wim Klein Haneveld, asset and liability management (Klein Haneveld et al. 2011), with Wim Klein Haneveld and Matthijs H. Streutker, as well as a joint project with Arno Maatman, Caspar Schweigman, and Arjan Ruijs on "Modeling farmers' response to uncertain rainfall in Burkina Faso: a stochastic programming approach" in Maatman et al. (2002). The intent and contribution of this project is described best by the following quotation from the paper, "This study draws important policy conclusions regarding reorientation of research programs and illustrates how operations research techniques can be usefully applied to study grass root problems in developing countries."

In addition to his research contributions, Maarten continually provided leadership, developing the Stochastic Programming Bibliography early in his career, serving as Secretary and then Chair of the Committee on Stochastic Programming, and serving as Program Director for the Bachelor of Science in Econometrics \& Operations Research and for the Master of Science in Econometrics, Operations Research \& Actuarial Studies at the University of Groningen. Maarten was an excellent teacher, winning the Lecturer of the Year Award in 2014 from the University of Groningen's Faculty of Economics and Business. Maarten cared deeply about those in his communities, and had a strong sense of integrity and social justice, advocating for the homeless when he was still in high school and holding to important principles without fail. Apart from beautiful mathematics Maarten enjoyed many other good things in life, which he happily shared with his friends and colleagues: music, in particular Bach, and tennis, which he made a fixed point of the social program of any stochastic programming meeting. We will miss Maarten's boisterous laugh, his warm smile, his sense of humor, and his ability to make everyone around him a better person.

\section{This special issue}

Maarten's articles have inspired, and will continue to inspire, contemporary research in stochastic (mixed-integer) optimization. This is illustrated by the research laid out in the present special issue. The lead article is a paper that Maarten van der Vlerk worked on with Niels van der Laan and Ward Romeijnders, improving Maarten's original error bounds on convex approximations for simple integer recourse models. The remaining articles are grouped according to Maarten's research interests: exploring structures, designing algorithms, and solving practical applications.

\subsection{Structures}

Weijun Xie and Shabbir Ahmed study a distributionally robust version of the simple integer recourse model introduced by Maarten, and they derive a closed form analytical expression for the objective and represent it by a mixed-integer second-order cone program. Lars Hellemo, Paul Barton, and Asgeir Tomasgard propose several formulations of stochastic programs with decision-dependent uncertainties and present a taxonomy of such problems. Vit Prochazka and Stein W. Wallace show how to save substantially 
on the number of scenarios to be evaluated for exactly computing the function values in stochastic programs. Matthias Claus, Rüdiger Schultz, and Kai Spürkel identify verifiable sufficient conditions for strong convexity of objective functions in risk-averse two-stage stochastic programs.

\subsection{Algorithms}

Andy Philpott, Vitor L. de Matos, and Lea Kapelevich develop a new variant of the stochastic dual dynamic programming algorithm to handle a data-driven distributionally robust formulation, with application to hydroelectric scheduling. Eli Towle and James Luedtke propose two new methods to solve the maximum-reliability stochastic network interdiction problem (SNIP) based on a compact deterministic equivalent formulation and a path-based formulation of SNIP, respectively. For dynamic mixed 0-1 optimization problems with strategic and operational stochastic parameters Laureano F. Escudero and Juan F. Monge present a modeling framework for risk neutral and risk averse approaches and outline a solution procedure based on nested stochastic decomposition. Semih Atakan and Suvrajeet Sen present a new framework allowing for effective utilization of Progressive Hedging guaranteeing convergence to globally optimal solutions of mixed-integer stochastic convex programs.

\subsection{Applications}

Jing Voon Chen, Julia L. Higle, and Michael Hintlian formulate a problem for model calibration of a Markov chain governing disease progression via a constrained optimization model, where the constraints capture model validity, probabilistic knowledge, clinical judgement, and more. Bismark Singh, David P. Morton, and Surya Santoso model integration of a conventional electric power generator and a wind farm, whose output is uncertain, in the context of a day-ahead electricity market. Miguel A. Lejeune and Janne Kettunen propose a new fractional stochastic integer programming model for forestry revenue management that includes a joint chance constraint with multirow random technology matrix and a joint integrated chance constraint. Giorgio Consigli, Vittorio Moriggia, Sebastiano Vitali, and Lorenzo Mercuri model an asset-liability management problem of a property and casualty (PC) company using a dynamic stochastic program and they analyze the relationship between risk capital constraints and risk-adjusted performance of a PC portfolio.

\section{References}

Albareda-Sambola M, van der Vlerk MH, Fernández E (2006) Exact solutions to a class of stochastic generalized assignment problems. Eur J Oper Res 173:465-487

Cremers MLAG, Klein Haneveld WK, van der Vlerk MH (2009) A two-stage model for a day-ahead paratransit planning problem. Math Methods Oper Res 69:323-341

Klein Haneveld WK, van der Vlerk MH (2006) Integrated chance constraints: reduced forms and an algorithm. Comput Manag Sci 3:245-269

Klein Haneveld WK, Stougie L, van der Vlerk MH (1995) On the convex hull of the simple integer recourse objective function. Ann Oper Res 56:209-224 
Klein Haneveld WK, Stougie L, van der Vlerk MH (1996) An algorithm for the construction of the convex hull of the simple integer recourse objective function. Ann Oper Res 64:67-81

Klein Haneveld WK, Stougie L, van der Vlerk MH (2006) Simple integer recourse models: convexity and convex approximations. Math Program 108:435-473

Klein Haneveld WK, Streutker MH, van der Vlerk MH (2011) Collective adjustment of pension rights in ALM models. Comput Manag Sci 8:137-156

Louveaux F, van der Vlerk MH (1993) Stochastic programming with simple integer recourse. Math Program 61:301-325

Maatman A, Schweigman C, Ruijs A, van der Vlerk MH (2002) Modeling farmers' response to uncertain rainfall in Burkina Faso: a stochastic programming approach. Oper Res 50:399-414

Romeijnders W, van der Vlerk MH, Klein Haneveld WK (2015) Convex approximations for totally unimodular integer recourse models: a uniform error bound. SIAM J Optim 25:130-158

Romeijnders W, Schultz R, van der Vlerk MH, Klein Haneveld WK (2016a) A convex approximation for two-stage mixed-integer recourse models with a uniform error bound. SIAM J Optim 26:426-447

Romeijnders W, van der Vlerk MH, Klein Haneveld WK (2016b) Total variation bounds on the expectation of periodic functions with applications to recourse approximations. Math Program 157:3-46

Romeijnders W, Morton DP, van der Vlerk MH (2017) Assessing the quality of convex approximations for two-stage totally unimodular integer recourse models. INFORMS J Comput 29:211-231

Schultz R, Stougie L, van der Vlerk MH (1998) Solving stochastic integer programs by enumeration: a framework using Gröbner basis reductions. Math Program 83:229-252

Tomasgard A, Audestad JA, Dye S, Stougie L, van der Vlerk MH, Wallace SW (1998) Modelling aspects of distributed processing in telecommunication networks. Ann Oper Res 82:161-184

van der Vlerk MH (1995) Stochastic programming with integer recourse, Thesis Rijksuniversiteit Groningen, Theses on Systems, Organisations, and Management. Labyrint Publications, Capelle a/d Ijssel

van der Vlerk MH (2004) Convex approximations for complete integer recourse models. Math Program 99:297-310

van der Vlerk MH (2010) Convex approximations for a class of mixed-integer recourse models. Ann Oper Res 177:139-150

\section{Affiliations}

\section{David P. Morton ${ }^{1} \cdot$ Ward Romeijnders ${ }^{2} \cdot$ Rüdiger Schultz ${ }^{3} \cdot$ Leen Stougie $^{4,5,6}$}

David P. Morton

david.morton@northwestern.edu

Ward Romeijnders

w.romeijnders@rug.nl

Leen Stougie

stougie@cwi.nl

1 Department of Industrial Engineering and Management Sciences, Northwestern University, 2145 Sheridan Road, Evanston, IL 60208-3119, USA

2 Department of Operations, Faculty of Economics and Business, University of Groningen, Nettelbosje 2, 9747 AE Groningen, The Netherlands

3 Faculty of Mathematics, University of Duisburg-Essen, Thea-Leymann-Str. 9, 45127 Essen, Germany

4 CWI, Science Park 123, 1098 XG Amsterdam, The Netherlands

5 INRIA-Erable, Amsterdam, The Netherlands

6 Vrije Universiteit, Amsterdam, The Netherlands 